\title{
Nonlinear Radial Consolidation Analysis of Soft Soil with Vertical Drains under Cyclic Loadings
}

\author{
Pyol Kim ${ }^{D},{ }^{1}$ Hyong-Sik Kim, ${ }^{1}$ Yong-Gun Kim, ${ }^{2}$ Chung-Hyok Paek, ${ }^{1}$ Song-Nam Oh, \\ Sung-Ryol So, ${ }^{3}$ and Chang-Son $\mathrm{Ok}^{4}$ \\ ${ }^{1}$ Faculty of Geology, Kim Il Sung University, Pyongyang, Democratic People's Republic of Korea \\ ${ }^{2}$ Faculty of Global Environmental Science, Kim Il Sung University, Pyongyang, Democratic People's Republic of Korea \\ ${ }^{3}$ Institute of Advanced Science, Kim Il Sung University, Pyongyang, Democratic People's Republic of Korea \\ ${ }^{4}$ Faculty of Energy Science, Kim Il Sung University, Pyongyang, Democratic People's Republic of Korea
}

Correspondence should be addressed to Pyol Kim; 18846415254@163.com

Received 25 March 2020; Revised 10 June 2020; Accepted 15 June 2020; Published 30 June 2020

Academic Editor: Fabio Rizzo

Copyright (c) 2020 Pyol Kim et al. This is an open access article distributed under the Creative Commons Attribution License, which permits unrestricted use, distribution, and reproduction in any medium, provided the original work is properly cited.

This paper presents analytical solutions for nonlinear radial consolidation of soft soil with vertical drains under various cyclic loadings. By considering the nonlinear variations of compressibility and permeability expressed by the logarithm relations $\left(e-\log \sigma^{\prime}\right.$ and $\left.e-\log k_{h}\right)$, the governing equation for nonlinear radial consolidation of the soil under equal strain and timedependent loading is established. The analytical solutions are derived for nonlinear radial consolidation under haversine cyclic loading, trapezoidal cyclic loading, rectangular cyclic loading, and triangular cyclic loading. The presented solution is verified through the degeneration into the existing solutions for nonlinear radial consolidation under constant and ramp loadings, which shows the solution proposed in this paper is more general for nonlinear radial consolidation under time-dependent loading. The nonlinear radial consolidation behavior of the soil with vertical drains subjected to various cyclic loadings is investigated using the solutions developed. The proposed solutions can be effectively utilized in the analysis of nonlinear radial consolidation under various cyclic loadings.

\section{Introduction}

In most coastal and marine areas, the soils beneath many structures are highly compressible and may be subjected to complicated cyclic loadings, such as filling and discharging in silos, tanks, and reservoirs, traffic loads on embankments, highways, railways, or airports, wave and wind actions for offshore structures, pile construction, and machine vibrations. The consolidation behavior of the soft soil under cyclic loadings is very complex and has been studied for many years. In the past decades, a lot of scholars have conducted research on one-dimensional (1D) consolidation of soft soil subjected to cyclic loadings based on either the linear consolidation theory [1-6] or the nonlinear consolidation theory [7-16]. It is worth mentioning that Razouki and Schanz [4] and Razouki et al. [5] studied 1D consolidation of a clay layer subjected to haversine cyclic loading with and without the rest period on the basis of linear assumptions. More recently, Kim et al. [16] derived analytical solutions for 1D nonlinear consolidation of a saturated soil with variable compressibility and permeability under various cyclic loadings. On the contrary, Kim et al. [17] presented analytical solutions for 1D consolidation of unsaturated soils under cyclic loadings.

Vertical drains are widely used for improvement of soft soil, and the radial consolidation behavior of the soil with vertical drains under cyclic loadings has also been investigated by many researchers. Indraratna et al. [18] conducted a large-scale triaxial test to examine the effectiveness of a prefabricated vertical drain (PVD) installed in soft clay subjected to cyclic loads, while $\mathrm{Ni}$ et al. $[19,20]$ proposed new constitutive models to predict the radial consolidation of soft soils under cyclic loading. Razouki [21] studied radial consolidation behavior of clay soil with vertical drains under 
free-strain condition and haversine cyclic loading based on the assumption that the consolidation coefficient in the radial direction is constant during the consolidation process.

Although there are many studies on 1D consolidation of the soil under cyclic loadings, research studies on radial consolidation under cyclic loadings are very limited, and any analytical solutions have hardly ever been proposed for nonlinear radial consolidation of the soil with vertical drains subjected to cyclic loadings with the consideration of variable compressibility and permeability. Moreover, the effects of different parameters on nonlinear radial consolidation under cyclic loadings have not been investigated. Therefore, this study is aimed to derive analytical solutions for nonlinear radial consolidation under various cyclic loadings and investigate nonlinear radial consolidation behavior of the soil under various cyclic loadings.

This paper is organized as follows. Firstly, the mathematical model and the governing equation for nonlinear radial consolidation of the soil with vertical drains subjected to time-dependent loading are presented in Section 2. Secondly, analytical solutions for nonlinear radial consolidation under cyclic loadings are derived in Section 3, and the proposed solutions are verified through the degeneration into special cases for nonlinear radial consolidation in Section 4. Thirdly, the effects of different parameters on nonlinear radial consolidation behavior of the soft soil with vertical drains under various cyclic loadings are investigated in Section 5. Finally, conclusions are given in Section 6.

\section{Mathematical Model and Governing Equation}

The mathematical model for radial consolidation analysis of soft soil with vertical drains is shown in Figure 1. Figures 1(a) and 1(b) show the arrangement of vertical drains and the cylindrical unit cell of the soil with a vertical drain, where $r_{w}, r_{s}$, and $r_{e}$ are the radii of the vertical drain, the smear zone, and the influence zone, respectively. The parameters $s, k_{s}$, and $k_{h}$ are the spacing of vertical drains and the horizontal permeability coefficients of the soil within the smear zone and the influence zone. $q(t)$ is the uniformly distributed time-dependent loading applied on the top surface of the soil of thickness $H$. In order to obtain the analytical solution for nonlinear radial consolidation of the soil with vertical drains subjected to time-dependent loading, the main assumptions are summarized as follows:

(1) The soil layer is saturated and homogeneous.

(2) The soil particles and pore water are incompressible.

(3) Only the radial flow of pore water is considered. Darcy's law is valid for the water flow.

(4) Both the loading and the settlement occur only in the vertical direction, while the soil and the vertical drain have an equal strain at any depth (i.e., the equal strain condition).

(5) The effect of the drain resistance is neglected.

(6) The nonlinear variations of compressibility and permeability during the consolidation process are given by

$$
\begin{gathered}
e=e_{0}-C_{c} \log \left(\frac{\sigma^{\prime}}{\sigma_{0}^{\prime}}\right), \\
e=e_{0}+C_{k} \log \left(\frac{k_{h}}{k_{h 0}}\right),
\end{gathered}
$$

where $e$ and $e_{0}$ are the void ratio at any time and the initial void ratio, respectively; $\sigma^{\prime}$ and $\sigma_{0}^{\prime}$ are the effective stress at any time and the initial effective stress, respectively; $k_{h}$ and $k_{h 0}$ are the horizontal permeability coefficients of the soil within the influence zone at any time and at the initial time, respectively; $C_{c}$ and $C_{k}$ are the compression index and the permeability index, respectively.

Based on equations (1) and (2), the following relations are obtained:

$$
\begin{gathered}
k_{h}=k_{h 0}\left(\frac{\sigma_{0}^{\prime}}{\sigma^{\prime}}\right)^{C_{c} / C_{k}} \\
m_{v}=-\frac{1}{1+e_{0}} \frac{\partial e}{\partial \sigma^{\prime}}=\frac{1}{1+e_{0}} \frac{C_{c}}{\ln 10} \frac{1}{\sigma^{\prime}}=m_{v 0}\left(\frac{\sigma_{0}^{\prime}}{\sigma^{\prime}}\right),
\end{gathered}
$$

where $m_{v}$ and $m_{v 0}$ are the compressibility coefficients of the soil at any time and at the initial time, respectively.

The governing equation for radial consolidation of the soil under the equal strain condition can be written as follows [22]:

$$
\left\{\begin{array}{cc}
\frac{2 r}{r_{s}^{2}-r^{2}} \cdot \frac{k_{s}}{\gamma_{w}} \cdot \frac{\partial u_{s}}{\partial r}=\frac{\partial \varepsilon}{\partial t}, & r_{w}<r<r_{s} \\
\frac{2 r}{r_{e}^{2}-r^{2}} \cdot \frac{k_{h}}{\gamma_{w}} \cdot \frac{\partial u_{n}}{\partial r}=\frac{\partial \varepsilon}{\partial t}, & r_{s}<r<r_{e}
\end{array}\right.
$$

where $\gamma_{w}$ is the unit weight of water; $u_{s}$ and $u_{n}$ are the excess pore water pressure within the smear zone and the influence zone, respectively; $\varepsilon$ is the vertical strain of the soil and the vertical drain; $r$ is the radial distance from the center of the vertical drain; $t$ is the time.

The average excess pore water pressure for both the smear zone and the influence zone, $\bar{u}$, can be defined as

$$
\bar{u}=\frac{1}{\pi\left(r_{e}^{2}-r_{w}^{2}\right)}\left(\int_{r_{w}}^{r_{s}} u_{s} 2 \pi r \mathrm{~d} r+\int_{r_{s}}^{r_{e}} u_{n} 2 \pi r \mathrm{~d} r\right) .
$$
by
The boundary conditions in the radial direction are given 


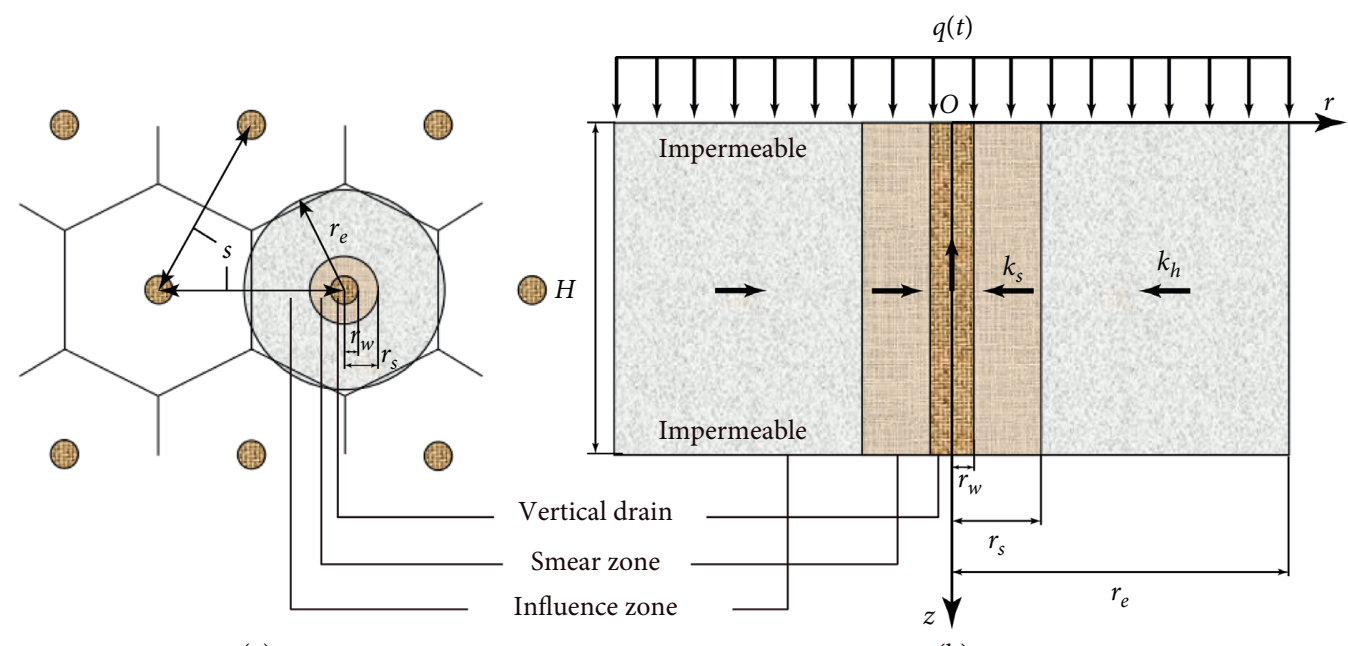

(a)

(b)

FIgURE 1: A schematic diagram for radial consolidation analysis. (a) The arrangement of vertical drains; (b) the cylindrical unit cell of the soil with a vertical drain.

$$
\begin{cases}u_{s}=0, & r=r_{w}, \\ u_{s}=u_{n}, & r=r_{s}, \\ k_{s} \frac{\partial u_{s}}{\partial r}=k_{h} \frac{\partial u_{n}}{\partial r}, & r=r_{s}, \\ \frac{\partial u_{n}}{\partial r}=0, & r=r_{e} .\end{cases}
$$

The initial condition for the average excess pore water pressure can be expressed as

$$
\bar{u}(r, 0)=q(0)=\bar{u}^{0} .
$$

\section{Analytical Solutions for Cyclic Loadings}

In this section, analytical solutions are derived for nonlinear radial consolidation of the soil with vertical drains under various cyclic loadings (Figure 2), such as haversine cyclic loading, trapezoidal cyclic loading, rectangular cyclic loading, and triangular cyclic loading.

3.1. Average Excess Pore Water Pressure. The excess pore water pressures within the smear zone and the influence zone can be obtained by integrating (5) and using the boundary conditions (7) as follows:

$$
\left\{\begin{array}{l}
u_{s}=\frac{\gamma_{w}}{2 k_{s}} \frac{\partial \varepsilon}{\partial t}\left(r_{e}^{2} \ln \frac{r}{r_{w}}-\frac{r^{2}-r_{w}^{2}}{2}\right), \quad r_{w}<r<r_{s}, \\
u_{n}=\frac{\gamma_{w}}{2 k_{h}} \frac{\partial \varepsilon}{\partial t}\left(r_{e}^{2} \ln \frac{r}{r_{s}}-\frac{r^{2}-r_{s}^{2}}{2}\right)+\frac{\gamma_{w}}{2 k_{s}} \frac{\partial \varepsilon}{\partial t}\left(r_{e}^{2} \ln \frac{r_{s}}{r_{w}}-\frac{r_{s}^{2}-r_{w}^{2}}{2}\right), \quad r_{s}<r<r_{e}
\end{array}\right.
$$

Substituting (9) into (6), the average excess pore water pressure can be determined by

$$
\bar{u}=\frac{r_{e}^{2} \xi_{n} \gamma_{w}}{2 k_{h}\left(1+e_{0}\right)} \frac{\partial e}{\partial \sigma^{\prime}}\left(\frac{\partial q}{\partial t}-\frac{\partial \bar{u}}{\partial t}\right)
$$

where

$$
\frac{\partial \varepsilon}{\partial t}=\frac{1}{\left(1+e_{0}\right)} \frac{\partial e}{\partial t}=\frac{1}{\left(1+e_{0}\right)} \frac{\partial e}{\partial \sigma^{\prime}} \frac{\partial \sigma^{\prime}}{\partial t}=\frac{1}{\left(1+e_{0}\right)} \frac{\partial e}{\partial \sigma^{\prime}}\left(\frac{\partial q}{\partial t}-\frac{\partial \bar{u}}{\partial t}\right),
$$

and $\xi_{n}$ is a parameter that reflects the characteristics of the smear zone and the influence zone of soil with vertical drains derived as follows:

$$
\xi_{n}=\frac{n^{2}}{n^{2}-1}\left[\ln (n)-\frac{3}{4}+\frac{4 n^{2}-1}{4 n^{2}}+\left(\frac{k_{h}}{k_{s}}-1\right)\left(\ln (m)+\frac{1-m^{2}}{n^{2}}+\frac{m^{4}-1}{4 n^{4}}\right)\right], \quad n=\frac{r_{e}}{r_{w}}, m=\frac{r_{s}}{r_{w}} .
$$




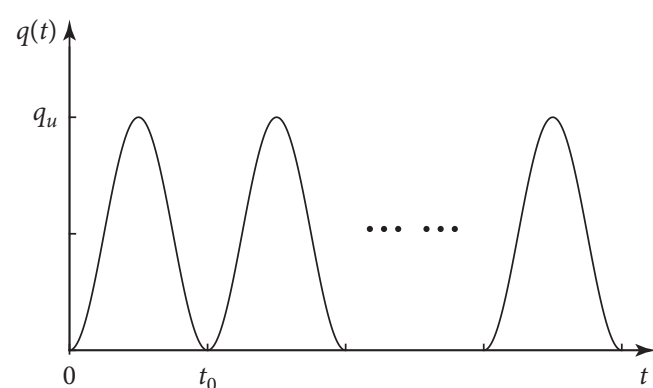

(a)

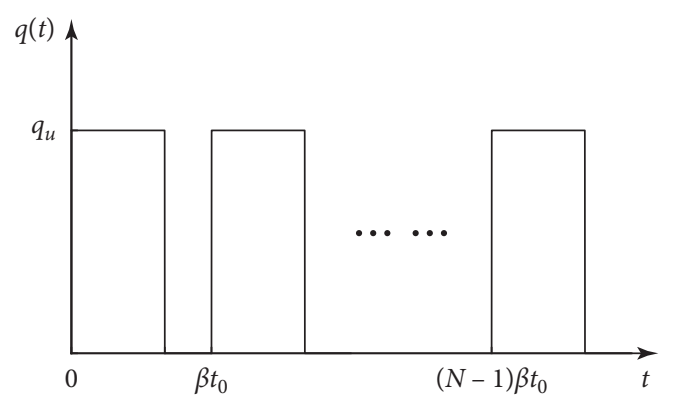

(c)

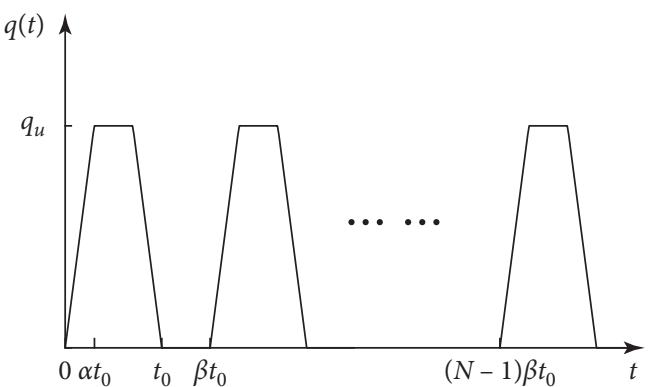

(b)

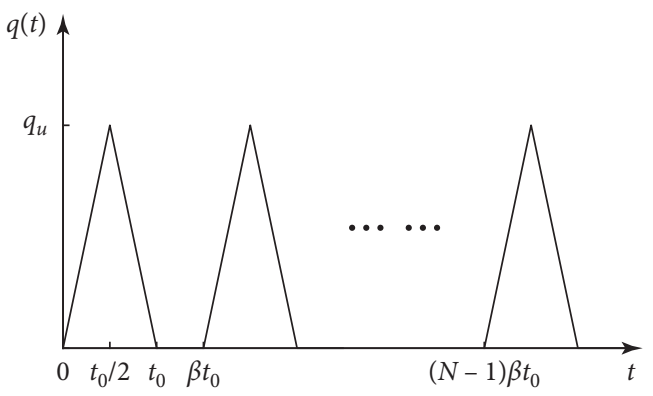

(d)

Figure 2: Various cyclic loadings. (a) Haversine cyclic loading; (b) trapezoidal cyclic loading; (c) rectangular cyclic loading; (d) triangular cyclic loading.

Defining the time factor in the radial direction $T_{h}=c_{h 0} t /\left(4 r_{e}^{2}\right)$, in which $c_{h 0}=k_{h 0}\left(1+e_{0}\right) /\left[\gamma_{w}\left(\partial e / \partial \sigma^{\prime}\right)_{t=0}\right]$ is the initial horizontal consolidation coefficient, and combining with equations (3), (4), and (10), the following expression can be obtained:

$$
\frac{\mathrm{d} \bar{u}}{\mathrm{~d} T_{h}}=-\frac{8}{\xi_{n}} \eta \bar{u}+\frac{\mathrm{d} q}{\mathrm{~d} T_{h}}
$$

where $\eta=\left(1+q-\bar{u} / \sigma_{0}^{\prime}\right)^{1-C_{c} / C_{k}}$.

Since (13) is a nonlinear ordinary differential equation, it is difficult to obtain its exact solution. $\eta$ varies from 1.0 to $\left(1+q_{u} / \sigma_{0}^{\prime}\right)^{\left(1-C_{c} / C_{k}\right)}$, in which $q_{u}$ is the ultimate value of loading $q$. Hence, $\eta$ in (13) can be assumed to be constant and have an average value given by $\bar{\eta}=1 / 2\left[1+\left(1+q_{u} / \sigma_{0}^{\prime}\right)^{\left(1-C_{c} / C_{k}\right)}\right]$ $[16,22,23]$. Then, (13) can be linearized to

$$
\frac{\mathrm{d} \bar{u}}{\mathrm{~d} T_{h}}=-\frac{8}{\xi_{n}} \overline{\eta u}+\frac{\mathrm{d} q}{\mathrm{~d} T_{h}} .
$$

The general solution to (14) satisfying the initial condition of (8) can be given as follows:

$$
\bar{u}=\exp \left(-\frac{8}{\xi_{n}} \bar{\eta} T_{h}\right)\left[\bar{u}^{0}+\int_{0}^{T_{h}} \frac{\mathrm{d} q}{\mathrm{~d} T_{h}} \exp \left(\frac{8}{\xi_{n}} \bar{\eta} \tau\right) \mathrm{d} \tau\right] .
$$

3.1.1. Haversine Cyclic Loading. Haversine cyclic loading shown in Figure 2(a) can be expressed as follows:

$$
q(t)=q_{u} \sin ^{2}\left(\frac{\pi t}{t_{0}}\right)
$$

where $t_{0}$ is the period of one loading cycle.

Substituting (16) into (15), the complete solution for the average excess pore water pressure under haversine cyclic loading can be obtained as follows:

$$
\bar{u}=\frac{\pi q_{u}}{\lambda^{2} T_{0}^{2}+4 \pi^{2}}\left\{\lambda T_{0} \sin \left(\frac{2 \pi T_{h}}{T_{0}}\right)-2 \pi\left[\cos \left(\frac{2 \pi T_{h}}{T_{0}}\right)-\exp \left(-\lambda T_{h}\right)\right]\right\},
$$

where $\quad \lambda=8 / \xi_{n} \cdot \bar{\eta}=4 / \xi_{n}\left(1+N_{\sigma}\right)^{1-C_{c} / C_{k}}, N_{\sigma}=\sigma_{0}^{\prime}+q_{u} /$ $\sigma^{\prime}$, and $T_{0}=c_{h 0} t_{0} / 4 r_{e}^{2}$.
3.1.2. Trapezoidal Cyclic Loading. Trapezoidal cyclic loading shown in Figure 2(b) can be expressed as follows: 


$$
q(t)=\left\{\begin{array}{l}
\frac{q_{u}}{\alpha t_{0}}\left[t-(N-1) \beta t_{0}\right], \quad(N-1) \beta t_{0} \leq t \leq[(N-1) \beta+\alpha] t_{0}, \\
q_{u}, \quad[(N-1) \beta+\alpha] t_{0} \leq t \leq[(N-1) \beta+(1-\alpha)] t_{0}, \\
-\frac{q_{u}}{\alpha t_{0}}\left[t-(N-1) \beta t_{0}-t_{0}\right], \quad[(N-1) \beta+(1-\alpha)] t_{0} \leq t \leq[(N-1) \beta+1] t_{0}, \\
0, \quad[(N-1) \beta+1] t_{0} \leq t \leq N \beta t_{0},
\end{array}\right.
$$

where $\beta t_{0}$ is the period of one loading cycle; $\alpha$ and $\beta$ are the loading parameters corresponding to the rate of loading increment or decrement and the rest period of loading, respectively; $N$ is the cycle number.

Similarly, substituting (18) into (17), the average excess pore water pressure for the trapezoidal cyclic loading can be written as

$\bar{u}= \begin{cases}\frac{q_{u} C_{1}}{\lambda T_{c}} \exp \left(-\lambda T_{h}\right), & (N-1) \beta t_{0} \leq t \leq[(N-1) \beta+\alpha] t_{0}, \\ \frac{q_{u} C_{2}}{\lambda T_{c}} \exp \left(-\lambda T_{h}\right), & {[(N-1) \beta+\alpha] t_{0} \leq t \leq[(N-1) \beta+(1-\alpha)] t_{0},} \\ \frac{q_{u} C_{3}}{\lambda T_{c}} \exp \left(-\lambda T_{h}\right), & {[(N-1) \beta+(1-\alpha)] t_{0} \leq t \leq[(N-1) \beta+1] t_{0},} \\ \frac{q_{u} C_{4}}{\lambda T_{c}} \exp \left(-\lambda T_{h}\right), & {[(N-1) \beta+1] t_{0} \leq t \leq N \beta t_{0},}\end{cases}$

where

$$
\begin{aligned}
C_{1} & =D(N-1)+D_{1}, \\
C_{2} & =D(N-1)+D_{2}, \\
C_{3} & =D(N-1)+D_{2}+D_{3}, \\
C_{4} & =D(N) \\
D(n) & =\sum_{N=1}^{n}\left(D_{2}+D_{4}\right), \\
D_{1} & =\exp \left(\lambda T_{h}\right)-\exp \left(\lambda T_{b}\right), \\
D_{2} & =\exp \left(\lambda T_{b}\right)\left[\exp \left(\lambda T_{c}\right)-1\right], \\
D_{3} & =\exp \left[\lambda\left(T_{f}-T_{c}\right)\right]-\exp \left(\lambda T_{h}\right), \\
D_{4} & =\exp \left(\lambda T_{f}\right)\left[\exp \left(-\lambda T_{c}\right)-1\right], \\
T_{c} & =\frac{c_{h 0} \alpha t_{0}}{\left(4 r_{e}^{2}\right)} \\
T_{b} & =\frac{c_{h 0}(N-1) \beta t_{0}}{4 r_{e}^{2}}, \\
T_{f} & =\frac{c_{h 0}[(N-1) \beta+1] t_{0}}{4 r_{e}^{2}} .
\end{aligned}
$$

3.1.3. Rectangular and Triangular Cyclic Loadings. Trapezoidal cyclic loading can be degenerated into rectangular cyclic loading and triangular cyclic loading by changing the value of the loading parameter $\alpha$. When $\alpha=0$, trapezoidal cyclic loading reduces into rectangular cyclic loading (Figure 2(c)). On the other hand, trapezoidal cyclic loading reduces to triangular cyclic loading (Figure 2(d)) when $\alpha=0.5$.

Thus, analytical solutions for the average excess pore water pressure under rectangular and triangular cyclic loadings can be obtained substituting $\alpha=0$ and $\alpha=0.5$ into (19), respectively.

3.2. Average Degree of Consolidation. Based on the average excess pore water pressures obtained under the above different cyclic loadings, the average degrees of consolidation with respect to excess pore water pressure and settlement, $U_{p}$ and $U_{s}$, can be expressed, respectively, as follows:

$$
\begin{gathered}
U_{p}=\frac{q-\bar{u}}{q_{u}}, \\
U_{s}=\frac{\int_{0}^{H} \varepsilon \mathrm{d} z}{\int_{0}^{H} \varepsilon_{f} \mathrm{~d} z}=a \frac{\int_{0}^{H} \log \left(\sigma^{\prime} / \sigma_{0}^{\prime}\right) \mathrm{d} z}{\int_{0}^{H} \log \left(\sigma_{f}^{\prime} / \sigma_{0}^{\prime}\right) \log \mathrm{d} z}=\frac{\ln \left(\sigma_{0}^{\prime}+q-\bar{u} / \sigma_{0}^{\prime}\right)}{\ln N_{\sigma}},
\end{gathered}
$$

where $\varepsilon_{f}$ and $\sigma_{f}^{\prime}$ are the final vertical strain and the final effective stress, respectively.

\section{Degeneration of the Proposed Solution to Special Cases}

It can be easily found that the proposed analytical solution for trapezoidal cyclic loading can be degenerated into the existing solutions for nonlinear radial consolidation under constant loading and ramp loading.

4.1. Constant Loading. When $\alpha=0, \beta=1$, and $N=1$, trapezoidal cyclic loading reduces to constant loading (Figure 3(a)) expressed by $q(t)=q_{u}$. Then, the solution for the average excess pore water pressure can be obtained as follows:

$$
\bar{u}=q_{u} \exp \left(-\lambda T_{h}\right)
$$




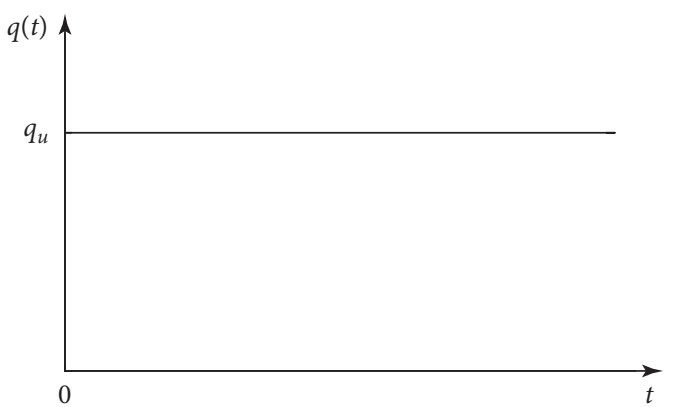

(a)

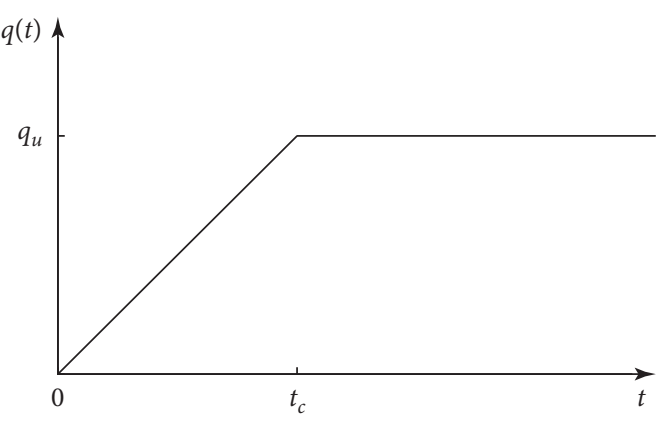

(b)

Figure 3: Constant loading (a) and ramp loading (b).

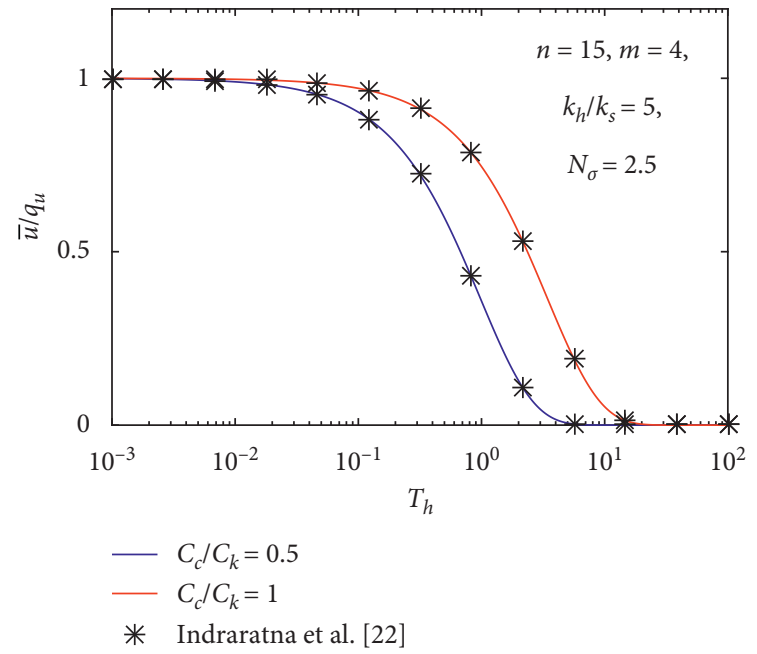

(a)

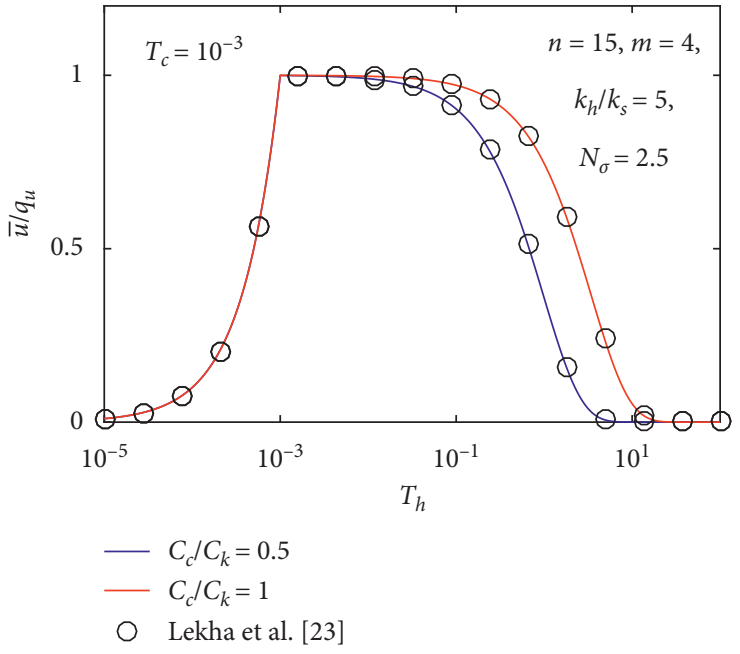

(b)

Figure 4: Comparison with the existing solutions for constant loading (a) and ramp loading (b).

Equation (23) is the solution for nonlinear radial consolidation of clays with variable permeability and compressibility under constant loading proposed by Indraratna et al. [22].

4.2. Ramp Loading. When $N=1$ and $t_{0}$ tends to infinity, trapezoidal cyclic loading reduces to ramp loading (Figure 3(b)) given by

$$
q(t)= \begin{cases}\frac{t}{t_{c}} q_{u}, & t \leq t_{c}, \\ q_{w}, & t \geq t_{c},\end{cases}
$$

where $t_{c}=\alpha t_{0}$.

The solution can be derived as

$$
\bar{u}=\left\{\begin{array}{l}
\frac{q_{u}}{\lambda T_{c}}\left[\exp \left(\lambda T_{h}\right)-1\right], \quad t \leq t_{c}, \\
\frac{q_{u}}{\lambda T_{c}} \exp \left(\lambda T_{h}\right)\left[1-\exp \left(-\lambda T_{c}\right)\right], \quad t \geq t_{c} .
\end{array}\right.
$$

Equation (25) is the solution for nonlinear radial consolidation of a clay layer with variable compressibility and permeability under ramp loading developed by Lekha et al. [23]. As shown in Figure 4, it can be seen that the results from the degenerate solutions and the existing solutions are consistent.

Through the degeneration mentioned above, it can be seen that the solution developed in this paper is a more general one for nonlinear radial consolidation under timedependent loading.

\section{Consolidation Behavior under Cyclic Loadings}

In this section, the effects of different parameters on nonlinear radial consolidation behavior under different types of cyclic loadings are investigated. Since the parameter $C_{c} / C_{k}$ mainly changes from 0.5 to 2.0 [24], $C_{c} / C_{k}=0.5$ and $C_{c} / C_{k}=1.5$ are taken for the cases of $C_{c} / C_{k}<1$ and $C_{c} / C_{k}>1$, respectively. The other properties adopted in this study are as follows: 

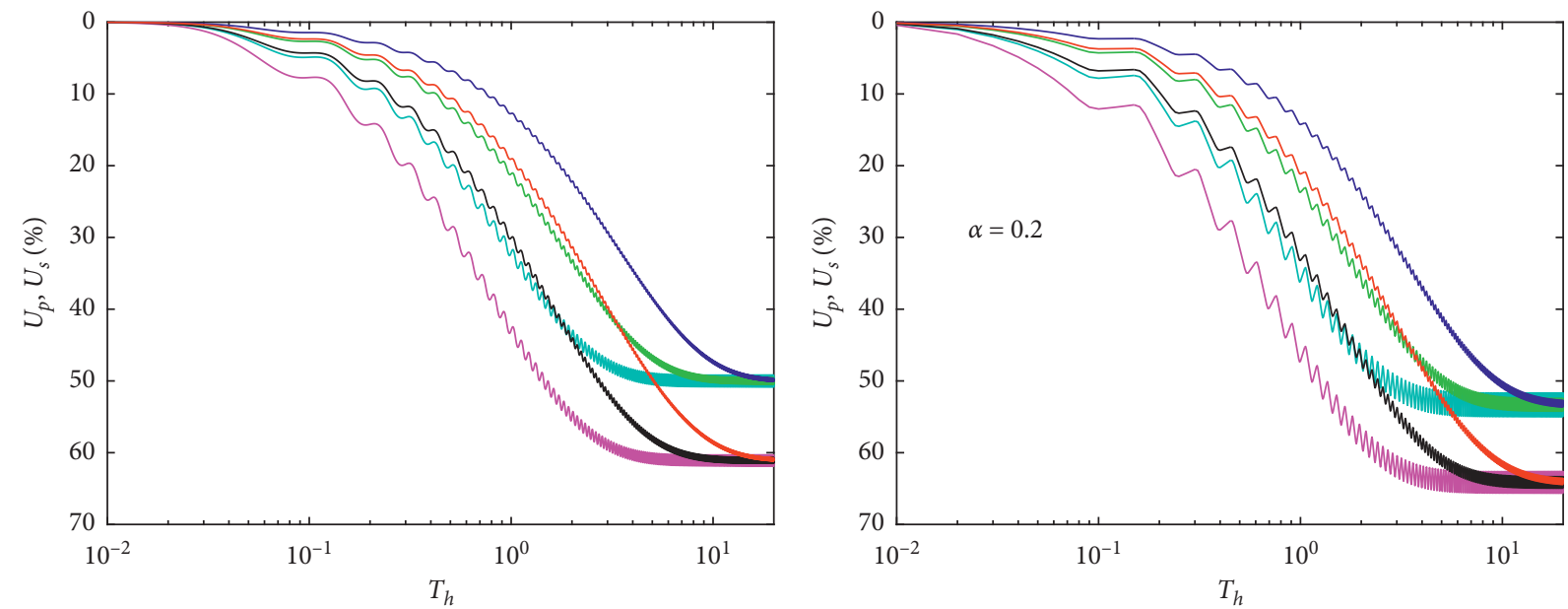

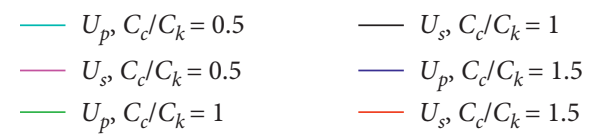

(a)

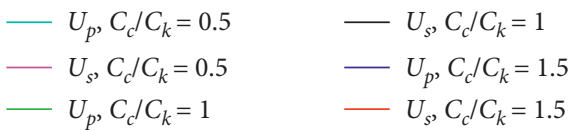

(b)

Figure 5: Nonlinear radial consolidation behavior at different values of $C_{c} / C_{k}$ under haversine cyclic loading (a) and trapezoidal cyclic loading (b).
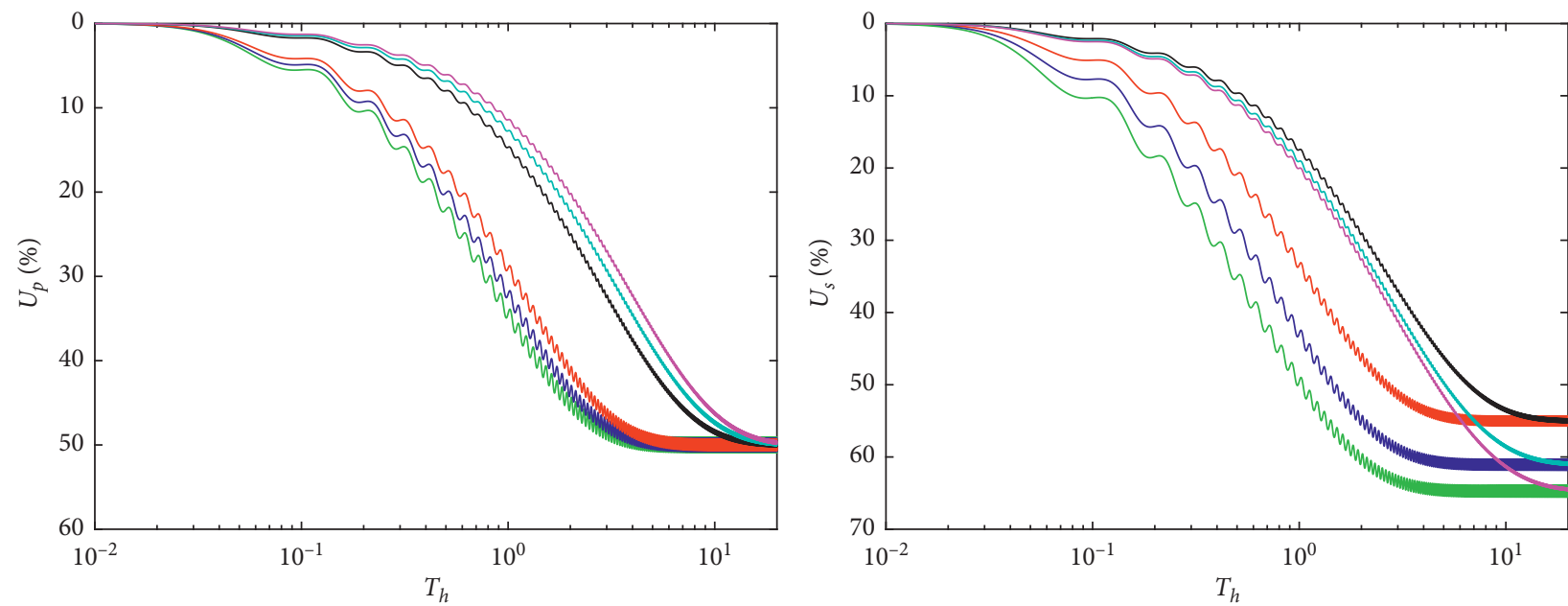
$C_{c} / C_{k}=0.5$
$C_{c} / C_{k}=1.5$
- $N_{\sigma}=1.5$
- $N_{\sigma}=1.5$
- $N_{\sigma}=2.5$
- $N_{\sigma}=2.5$
- $N_{\sigma}=3.5$
$-N_{\sigma}=3.5$

$\begin{array}{ll}C_{c} / C_{k}=0.5 & C_{c} / C_{k}=1.5 \\ -N_{\sigma}=1.5 & -N_{\sigma}=1.5 \\ -N_{\sigma}=2.5 & -N_{\sigma}=2.5 \\ -N_{\sigma}=3.5 & -N_{\sigma}=3.5\end{array}$

(a)

Figure 6: Continued. 

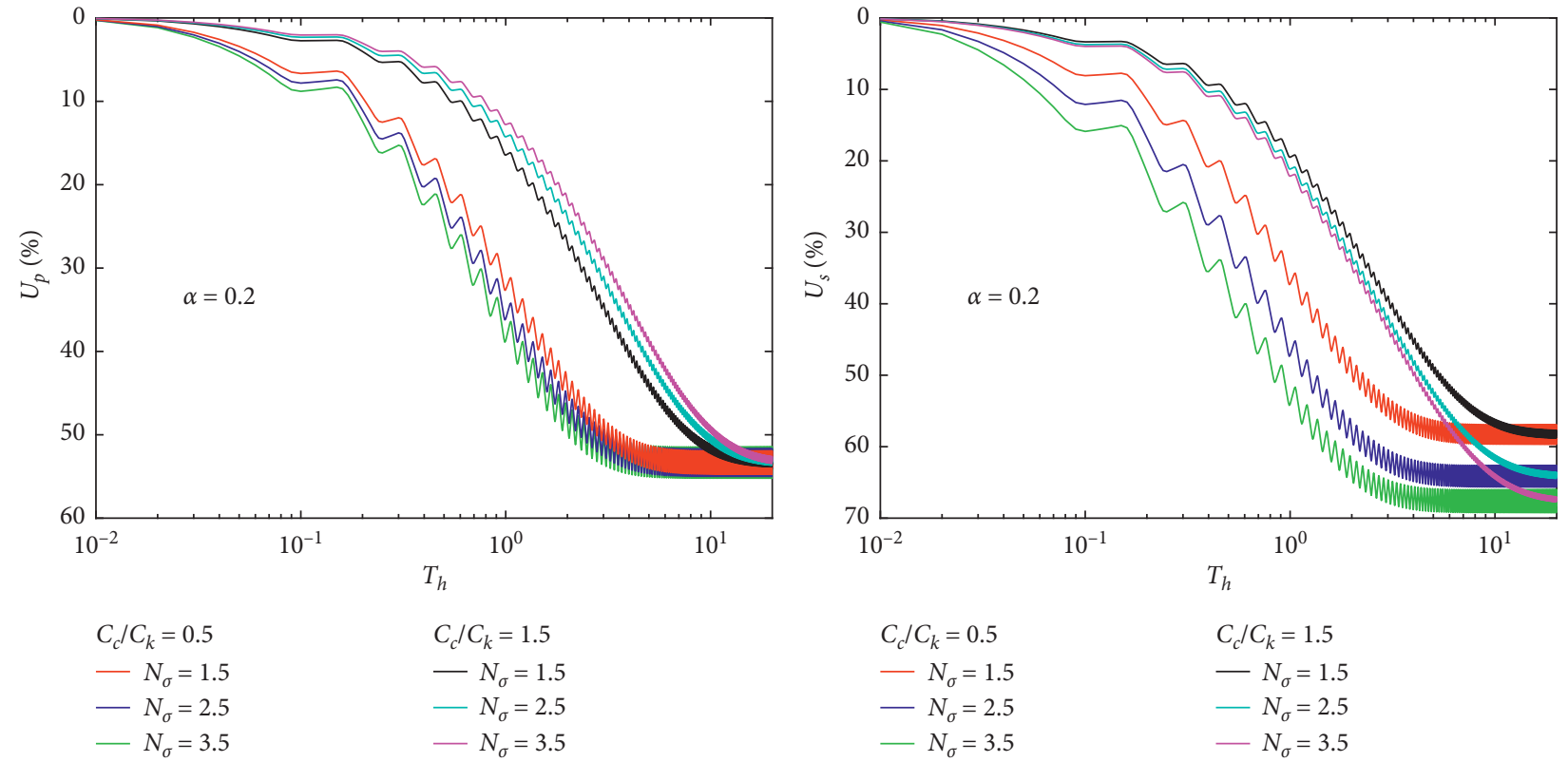

(b)

Figure 6: Nonlinear radial consolidation behavior at different values of $N_{\sigma}$ under haversine cyclic loading (a) and trapezoidal cyclic loading (b).
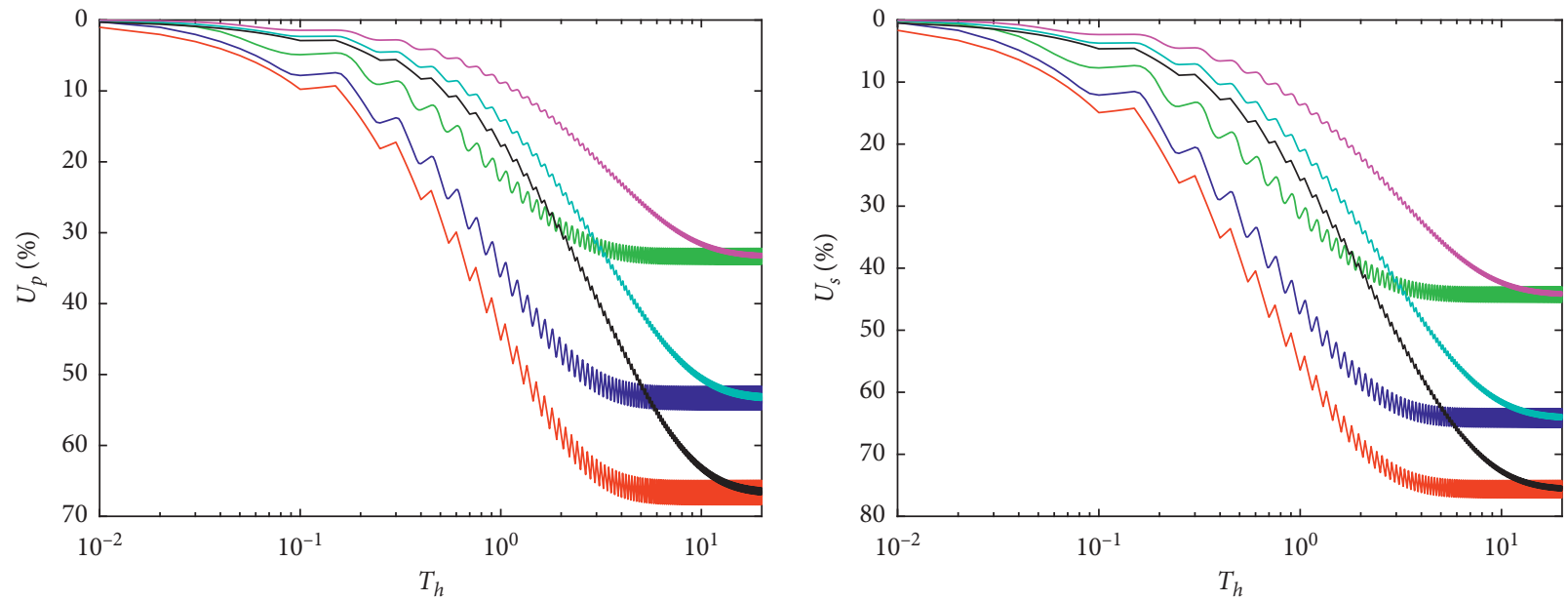
$C_{c} / C_{k}=0.5$
$C_{c} / C_{k}=1.5$
$-\alpha=0$
$-\alpha=0$
$-\alpha=0.2$
$-\alpha=0.2$
$-\alpha=0.5$
$-\alpha=0.5$

$C_{c} / C_{k}=0.5$

$C_{c} / C_{k}=1.5$

$-\alpha=0$

$-\alpha=0$

$-\alpha=0.2$

$\alpha=0.2$

FIGURE 7: Nonlinear radial consolidation behavior under cyclic loadings with different loading parameter $\alpha$.

$$
\begin{aligned}
n & =15, \\
m & =4, \\
\frac{k_{h}}{k_{s}} & =5, \\
N_{\sigma} & =2.5, \\
T_{0} & =0.1, \\
\beta & =1.5 .
\end{aligned}
$$

Figure 5 shows the variation of the average degree of consolidation $U_{p}$ and $U_{s}$ with time factor $T_{h}$ at different values of $C_{c} / C_{k}$ under different types of cyclic loadings. It can be found that average degree of consolidation $U_{p}$ and $U_{s}$ decreases but fluctuates with the increase in $C_{c} / C_{k}$, suggesting that the dissipation rate of excess pore water pressure and the settlement rate decrease with the fluctuation when $C_{c} / C_{k}$ increases. Furthermore, a bigger value of $C_{c} / C_{k}$ results in a smaller amplitude of fluctuation in the dissipation rate and the settlement rate. In addition, it can be seen that $U_{s}$ is 

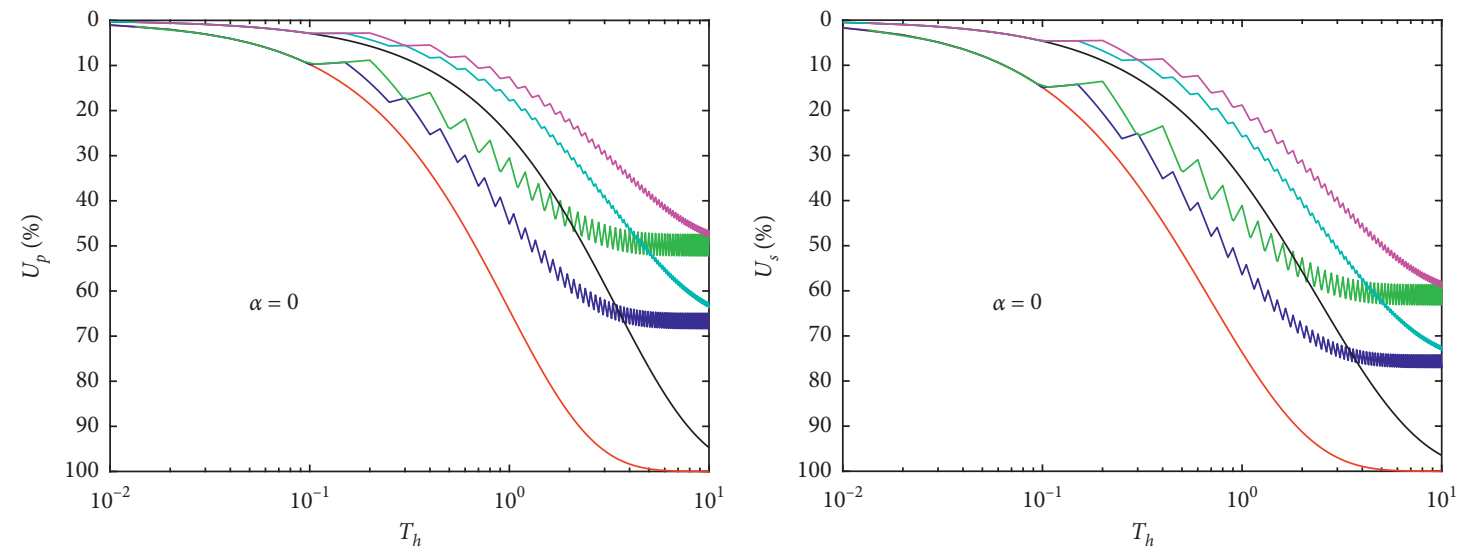
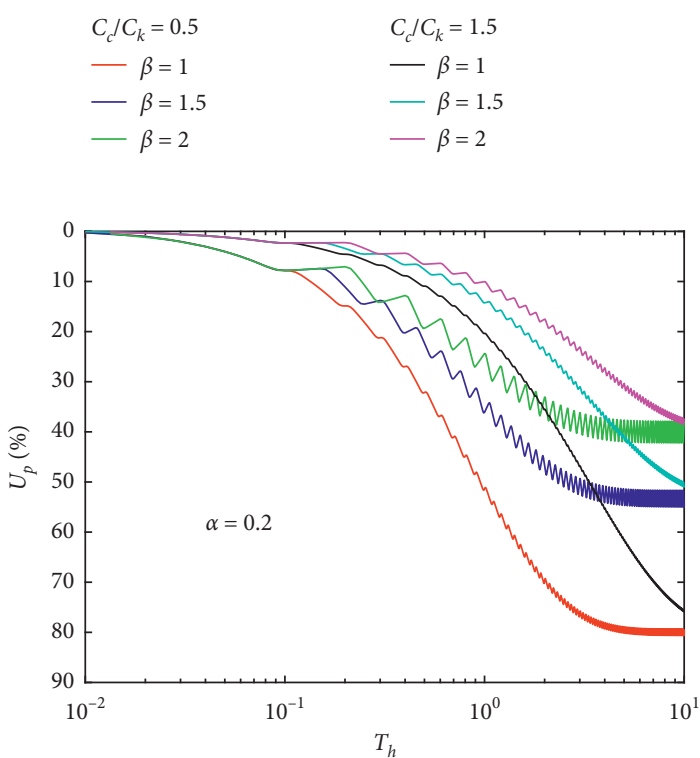
$C_{c} / C_{k}=0.5$
$-\beta=1$
$-\beta=1.5$
- $\beta=2$
$C_{c} / C_{k}=1.5$
$-\beta=1$
$-\beta=1.5$
$-\beta=2$

(a)

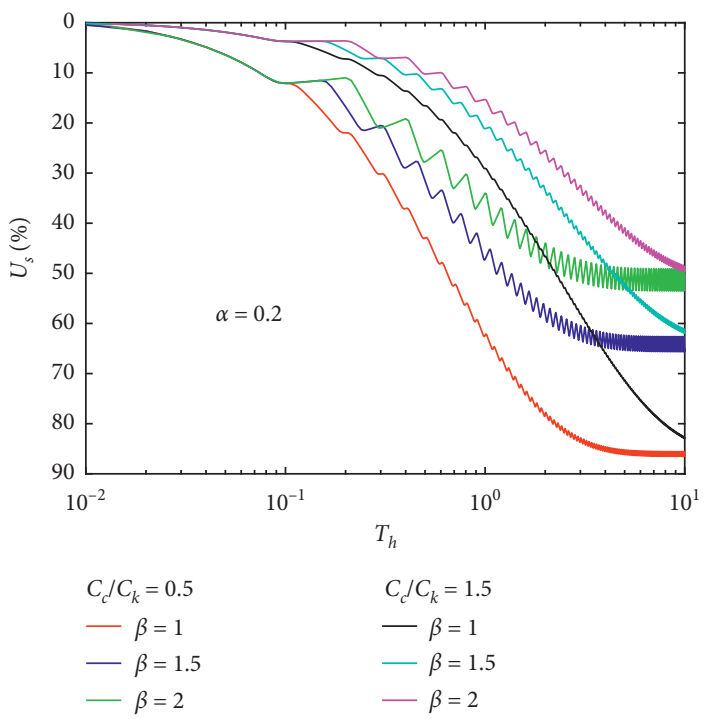

(b)
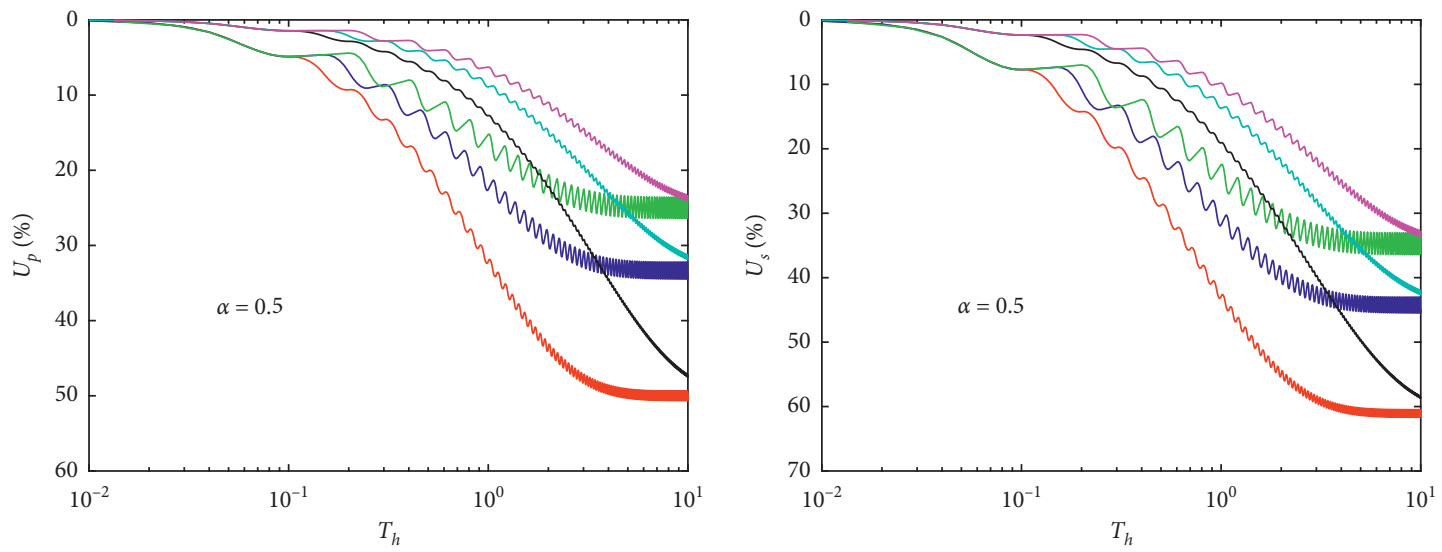
$C_{c} / C_{k}=0.5$
$C_{c} / C_{k}=1.5$
$-\beta=1$
$-\beta=1$
$-\beta=1.5$
$-\beta=2$
$-\beta=1.5$
- $\beta=2$
$C_{c} / C_{k}=0.5$
$C_{c} / C_{k}=1.5$
$-\beta=1$
$-\beta=1.5$
$-\beta=2$
$-\beta=1$
$-\beta=1.5$
$-\beta=2$

(c)

Figure 8: Nonlinear radial consolidation behavior under cyclic loadings with different loading parameter $\beta$. Rectangular cyclic loading (a); trapezoidal cyclic loading (b); triangular cyclic loading (c). 

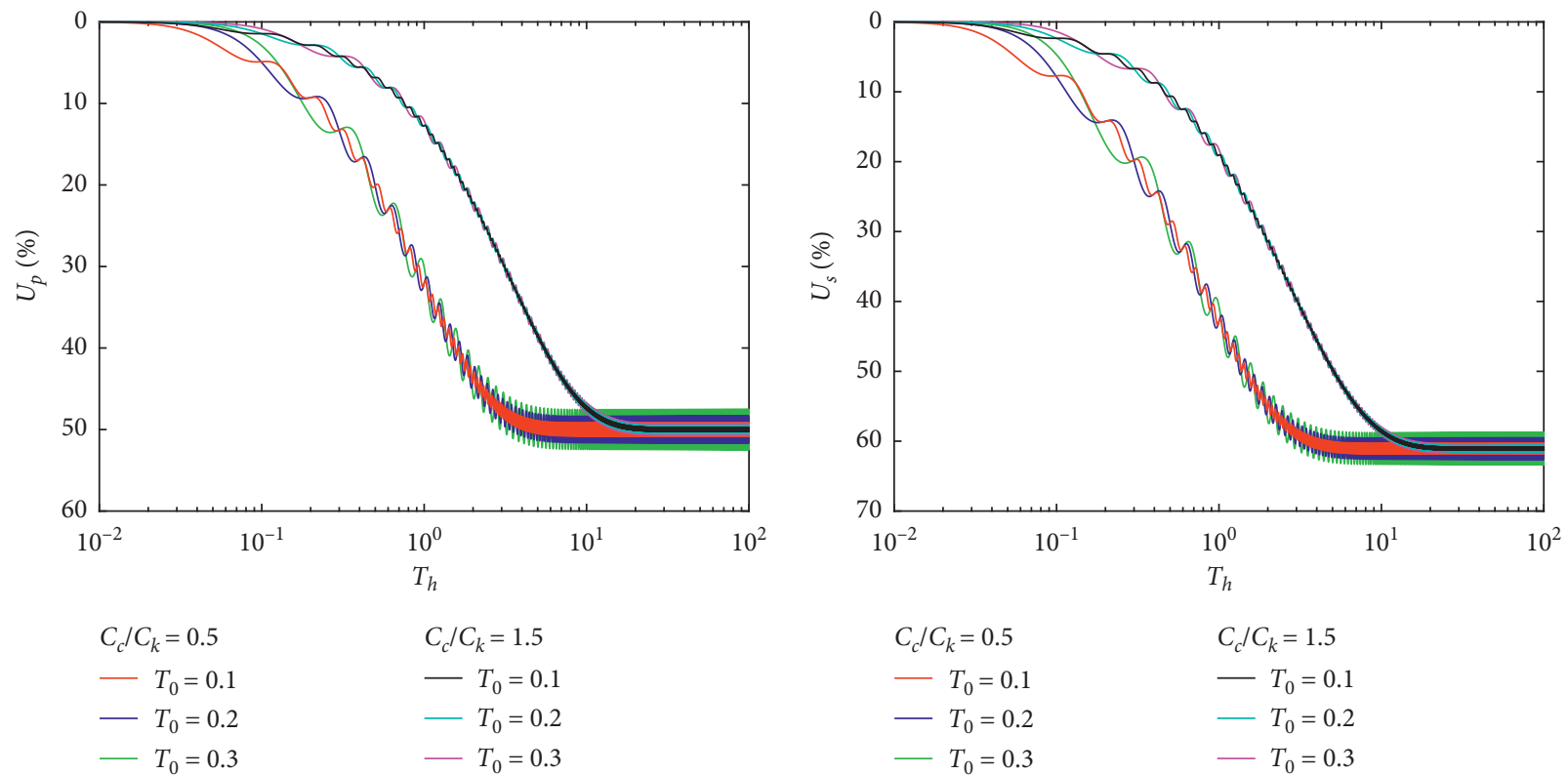

(a)
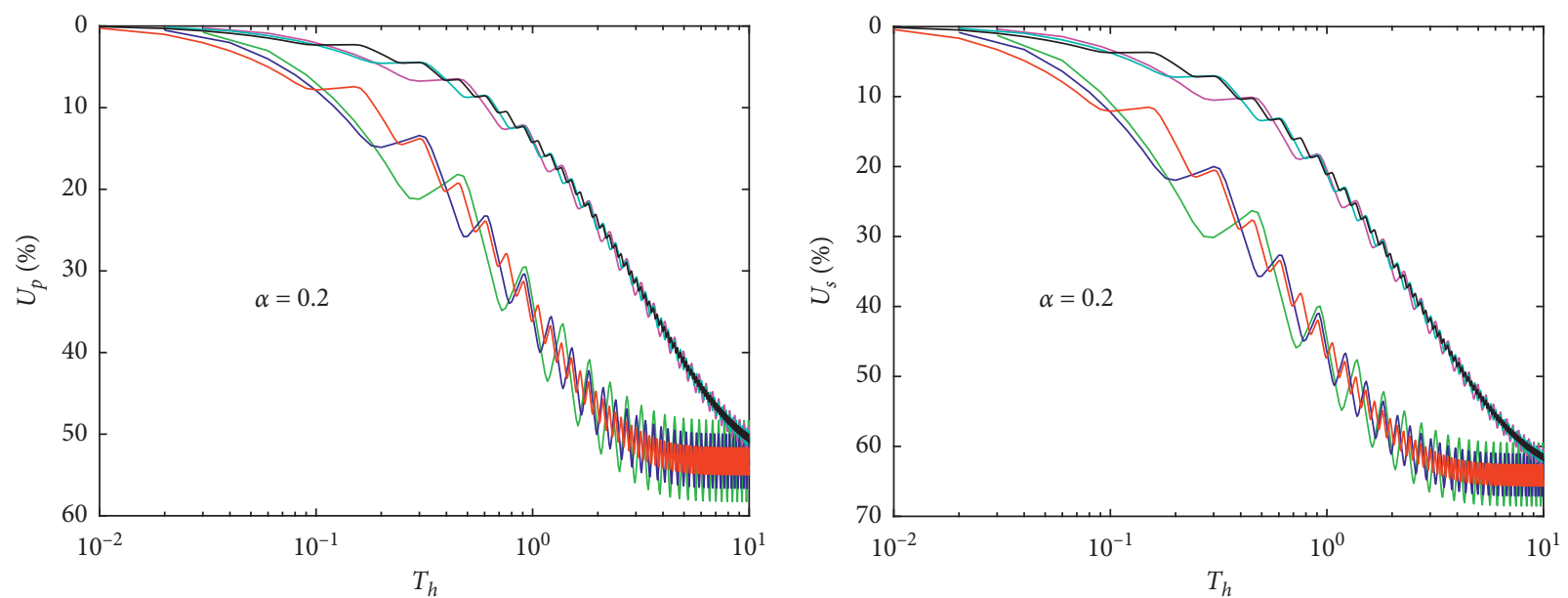
$C_{c} / C_{k}=0.5$
$C_{c} / C_{k}=1.5$
- $T_{0}=0.1$
- $T_{0}=0.1$
- $T_{0}=0.2$
- $T_{0}=0.2$
- $T_{0}=0.3$
$-T_{0}=0.3$
$C_{c} / C_{k}=0.5$
$C_{c} / C_{k}=1.5$
- $T_{0}=0.1$
- $T_{0}=0.1$
- $T_{0}=0.2$
- $T_{0}=0.2$
- $T_{0}=0.3$
$-T_{0}=0.3$

(b)

FiguRe 9: Nonlinear radial consolidation behavior under cyclic loadings with different loading parameter $T_{0}$ : haversine cyclic loading (a); trapezoidal cyclic loading (b).

always bigger than $U_{p}$ in all cases, which means the rate of settlement is faster than that of dissipation of excess pore water pressure during the consolidation process.

Figure 6 presents the variation of the average degree of consolidation $U_{p}$ and $U_{s}$ with time factor $T_{h}$ at different values of $N_{\sigma}$ under different types of cyclic loadings. It can be found that a bigger value of $N_{\sigma}$ results in a faster dissipation rate of excess pore water pressure in the case of $C_{c} / C_{k}<1$, but a smaller value of $N_{\sigma}$ leads to a faster dissipation rate in the case of $C_{c} / C_{k}>1$. Meanwhile, the bigger value of $N_{\sigma}$ increases the rate of settlement in all cases of
$C_{c} / C_{k}<1$ and $C_{c} / C_{k}>1$. Since $N_{\sigma}$ is related to the maximum loading $q_{u}$, the following conclusion is drawn: the dissipation rate increases when maximum loading $q_{u}$ increases in the case of $C_{c} / C_{k}<1$ but decreases with the increase in maximum loading $q_{u}$ in the case of $C_{c} / C_{k}>1$, whereas the settlement rate increases with the increase in maximum loading $q_{u}$ in all cases of $C_{c} / C_{k}<1$ and $C_{c} / C_{k}>1$.

Figure 7 represents the variation of the average degree of consolidation $U_{p}$ and $U_{s}$ with time factor $T_{h}$ under different types of cyclic loadings with different loading parameter $\alpha$. It can be seen that the smaller the loading parameter $\alpha$ is, the 
more quickly the dissipation and the settlement develop in all cases of $C_{c} / C_{k}<1$ and $C_{c} / C_{k}>1$. As the loading parameter $\alpha$ reflects the rate of loading increment or decrement, it can be found that the higher the rate of loading increment or decrement is, the faster the rates of dissipation and settlement are. Moreover, the rates of dissipation and settlement under rectangular cyclic loading (i.e., $\alpha=0$ ) are the biggest, and the rates of dissipation and settlement under triangular cyclic loading (i.e., $\alpha=0.5$ ) are the smallest.

Figure 8 describes the variation of the average degree of consolidation $U_{p}$ and $U_{s}$ with time factor $T_{h}$ under different cyclic loadings with different loading parameter $\beta$. As shown in Figure 8(a), through the results under rectangular cyclic loading $(\alpha=0)$ for different loading parameter $\beta$, it can be found that the rates of dissipation and settlement increase with the decrease in the value of loading parameter $\beta$ in all cases of $C_{c} / C_{k}<1$ and $C_{c} / C_{k}>1$. Moreover, rectangular cyclic loading without the rest period (i.e., $\beta=1$ ) reduces into constant loading, and the rates of dissipation and settlement are the highest and increase monotonically with time in that case. The results under trapezoidal cyclic loading and triangular cyclic loading for different loading parameter $\beta$ can be observed in Figures 8(b) and 8(c), respectively. Similar to the results in Figure 8(a), the dissipation rate and the settlement rate increase with the decrease in the value of loading parameter $\beta$ in all cases of $C_{c} / C_{k}<1$ and $C_{c} / C_{k}>1$. As well as, a smaller value of the loading parameter $\beta$ results in more cycles and a smaller amplitude of fluctuation in the rates of dissipation and settlement. Since the loading parameter $\beta$ reflects the rest period of loading, it is quite obvious that a longer rest period of loading induces a decrease in the rates of dissipation and settlement, less cycles, and a bigger amplitude of fluctuation.

Figure 9 outlines the variation of the average degree of consolidation $U_{p}$ and $U_{s}$ with time factor $T_{h}$ under different cyclic loadings with different loading parameter $T_{0}$. It can be seen that a smaller value of the loading parameter $T_{0}$ induces a smaller amplitude and more cycles of fluctuation in the rates of dissipation and settlement in all cases of $C_{c} / C_{k}<1$ and $C_{c} / C_{k}>1$. In addition, the loading parameter $T_{0}$ has a significant influence on the amplitude and the cycles of fluctuation in the dissipation rate and the settlement rate but does not affect the mean value of the rates. The loading parameter $T_{0}$ is related to the period of loading, and thus it can be found that the longer the period of loading, the bigger the amplitude and less cycles of fluctuation in the dissipation rate and the settlement rate.

It should be noted that unlike constant loading and ramp loading conditions, the dissipation process of the excess pore water pressure does not complete after a long time but rather continues oscillating with a certain amplitude, and the average degrees of consolidation under cyclic loadings do not reach $100 \%$ in all cases of $C_{c} / C_{k}<1$ and $C_{c} / C_{k}>1$. It is due to repetitive loading and unloading stages of cyclic loadings, which results in squeezing out of pore water during the loading stage and then absorbing during the unloading stage. Thus, neglecting the effect of cyclic loadings may result in overestimated settlement and cannot consider the continuous volume change.
It should also be pointed out that the present solution is only valid for the elastic constitutive model, which is not suitable for the elastic-plastic model of soft soil with creep. However, the analytical solution is first proposed for nonlinear radial consolidation of soft soil with vertical drains subjected to cyclic loadings. Also, the proposed solution is more general for the soil under time-dependent loadings and can be easily used by engineers.

\section{Conclusion}

In this paper, analytical solutions are derived for nonlinear radial consolidation of soft soil with vertical drains subjected to various cyclic loadings, such as haversine, trapezoidal, rectangular, and triangular cyclic loadings. The presented solution is verified through the degeneration into the existing solutions for nonlinear radial consolidation under constant and ramp loadings. The nonlinear radial consolidation behavior under various cyclic loadings is investigated. The results show that under cyclic loadings, both the dissipation rate of excess pore water pressure and the settlement rate slow down with the fluctuation corresponding to cyclic loadings when $C_{c} / C_{k}$ increases. The dissipation rate increases when maximum loading $q_{u}$ increases in the case of $C_{c} / C_{k}<1$ but decreases with the increase in the maximum loading $q_{u}$ in the case of $C_{c} / C_{k}>1$, whereas the settlement rate increases with the increase in the maximum loading $q_{u}$ in all cases of $C_{c} / C_{k}<1$ and $C_{c} / C_{k}>1$. The higher the rate of loading increment or decrement is, the faster the dissipation rate and the settlement rate are. A longer rest period of loading induces the decrease in the rates of dissipation and settlement, less cycles, and a bigger amplitude of fluctuation. The longer the period of loading, the bigger the amplitude and less cycles of fluctuation in the rates.

\section{Data Availability}

The data used to support the findings of this study are included within the article.

\section{Conflicts of Interest}

The authors declare that they have no known competing financial interests or personal relationships that could have appeared to influence the work reported in this paper.

\section{Acknowledgments}

This work was supported by Kim Il Sung University in DPR of Korea.

\section{References}

[1] N. E. Wilson and M. M. Elgohary, "Consolidation of soils under cyclic loading," Canadian Geotechnical Journal, vol. 11, no. 3, pp. 420-423, 1974.

[2] M. Favaretti and M. Soranzo, "A simplified consolidation theory in cyclic loading conditions," in Proceedings of the International Symposium on Compression and Consolidation of Clayey Soils, A. A. Balkema, Rotterdam, Netherlands, pp. 405-409, 1995. 
[3] E. Conte and A. Troncone, "One-dimensional consolidation under general time-dependent loading," Canadian Geotechnical Journal, vol. 43, no. 11, pp. 1107-1116, 2006.

[4] S. S. Razouki and T. Schanz, "One-dimensional consolidation under haversine repeated loading with rest period," Acta Geotechnica, vol. 6, no. 1, pp. 13-20, 2011.

[5] S. S. Razouki, P. Bonnier, M. Datcheva, and T. Schanz, "Analytical solution for $1 \mathrm{D}$ consolidation under haversine cyclic loading," International Journal for Numerical and Analytical Methods in Geomechanics, vol. 37, no. 14, pp. 2367-2372, 2013.

[6] P. Kim, Y.-G. Kim, C.-H. Paek, and J. Ma, "Lattice Boltzmann method for consolidation analysis of saturated clay," Journal of Ocean Engineering and Science, vol. 4, no. 3, pp. 193-202, 2019.

[7] M. M. Baligh and J. N. Levadoux, "Consolidation theory for cyclic loading," Journal of the Geotechnical Engineering Division, vol. 104, no. 4, pp. 415-431, 1978.

[8] E. H. Davis and G. P. Raymond, "A non-linear theory of consolidation,” Géotechnique, vol. 15, no. 2, pp. 161-173, 1965.

[9] K.-H. Xie, T. Qi, and Y.-Q. Dong, "Nonlinear analytical solution for one-dimensional consolidation of soft soil under cyclic loading," Journal of Zhejiang University-SCIENCE A, vol. 7, no. 8, pp. 1358-1364, 2006.

[10] X. Geng, C. Xu, and Y. Cai, "Non-linear consolidation analysis of soil with variable compressibility and permeability under cyclic loadings," International Journal for Numerical and Analytical Methods in Geomechanics, vol. 30, no. 8, pp. 803-821, 2006.

[11] Y.-Q. Cai, X.-Y. Geng, and C.-J. Xu, "Solution of one-dimensional finite-strain consolidation of soil with variable compressibility under cyclic loadings," Computers and Geotechnics, vol. 34, no. 1, pp. 31-40, 2007.

[12] E. Conte and A. Troncone, "Nonlinear consolidation of thin layers subjected to time-dependent loading," Canadian Geotechnical Journal, vol. 44, no. 6, pp. 717-725, 2007.

[13] M. M. Toufigh and A. Ouria, "Consolidation of inelastic clays under rectangular cyclic loading," Soil Dynamics and Earthquake Engineering, vol. 29, no. 2, pp. 356-363, 2009.

[14] D. Cheng, W. Wang, X. Chen, and Z. Zhang, "Finite analytic method for one-dimensional nonlinear consolidation under time-dependent loading," Shock and Vibration, vol. 2017, pp. 1-12, 2017.

[15] P. Kim, Y. G. Kim, H. B. Myong, C. H. Paek, and J. Ma, "Numerical analysis for nonlinear consolidation of saturated soil using lattice Boltzmann method," International Research Journal of Engineering and Technology, vol. 6, no. 4, pp. 3611-3618, 2019.

[16] P. Kim, K. S. Ri, Y. G. Kim, K. N. Sin, H. B. Myong, and C. H. Paek, "Nonlinear consolidation analysis of a saturated clay layer with variable compressibility and permeability under various cyclic loadings," International Journal of Geomechanics, vol. 20, no. 8, Article ID 04020111, 2020.

[17] P. Kim, M. Ri, Y. Kim, G. Ri, and H. Myong, "One-dimensional consolidation analysis of unsaturated soils under cyclic loadings," Shock and Vibration, vol. 2020, pp. 1-16, 2020.

[18] B. Indraratna, A. Attya, and C. Rujikiatkamjorn, "Experimental investigation on effectiveness of a vertical drain under cyclic loads," Journal of Geotechnical and Geoenvironmental Engineering, vol. 135, no. 6, pp. 835-839, 2009.

[19] J. Ni, B. Indraratna, X.-Y. Geng, J. P. Carter, and C. Rujikiatkamjorn, "Radial consolidation of soft soil under cyclic loads," Computers and Geotechnics, vol. 50, pp. 1-5, 2013.

[20] J. Ni, B. Indraratna, X.-Y. Geng, J. P. Carter, and Y.-L. Chen, "Model of soft soils under cyclic loading," International
Journal of Geomechanics, vol. 15, no. 4, Article ID 04014067, 2015.

[21] S. S. Razouki, "Radial consolidation clay behaviour under haversine cyclic load," Proceedings of the Institution of Civil Engineers - Ground Improvement, vol. 169, no. 2, pp. 143-149, 2016.

[22] B. Indraratna, C. Rujikiatkamjorn, and I. Sathananthan, "Radial consolidation of clay using compressibility indices and varying horizontal permeability," Canadian Geotechnical Journal, vol. 42, no. 5, pp. 1330-1341, 2005.

[23] K. R. Lekha, N. R. Krishnaswamy, and P. Basak, "Consolidation of clay by sand drain under time-dependent loading," Journal of Geotechnical and Geoenvironmental Engineering, vol. 124, no. 1, pp. 91-94, 1998.

[24] P. L. Berry and W. B. Wilkinson, "The radial consolidation of clay soils," Géotechnique, vol. 19, no. 2, pp. 253-284, 1969. 